\title{
Uptake and continuation rates of the intrauterine system in a university student general practice population in the UK
}

\author{
Catherine Marie Armitage, ${ }^{1}$ Carolyn Mitchell, ${ }^{2}$ Caroline Wigan, ${ }^{3}$ \\ Debbie A Smith ${ }^{4}$
}

${ }^{1}$ General Practitioner, Leeds Student Medical Practice, Leeds, UK

${ }^{2}$ Practice Nurse, Leeds Student Medical Practice, Leeds, UK ${ }^{3}$ Clinical Research Co-ordinator, Leeds Student Medical Practice, Leeds, UK

${ }^{4}$ General Practitioner, Leeds Student Medical Practice, Leeds, UK

\section{Correspondence to}

Dr Catherine Marie Armitage, Leeds Student Medical Practice, 4 Blenheim Court, Blenheim Walk, Leeds LS2 9AE, UK; catherine.armitage@nhs.net

Received 14 May 2012 Accepted 16 August 2012 Published Online First

14 December 2012
To cite: Armitage $\mathrm{CM}$, Mitchell C, Wigan C, et al. I Fam Plann Reprod Health Care 2013:39:186-189.

\begin{abstract}
Objective To follow a series of 100 women attending for fitting of the levonorgestrelreleasing intrauterine system (LNG IUS) registered at a single urban general practice serving the students of the local universities and higher education colleges.

Methods This was a prospective observational study. A questionnaire was completed by the fitter in discussion with the patient at the time of attendance for IUS fitting. Follow up was by telephone at 6 weeks, 6 months and 9-12 months after fitting.
\end{abstract}

Results The age range of women within the series was 18-38 years. 97 women were nulliparous. 37 women selected the IUS as their preferred method of contraception. 12 women chose the IUS primarily for non-contraceptive reasons. 75 women still had the IUS at 12 months' follow up with 11 lost to follow up at this point. No pregnancies or perforations were reported.

Conclusions The IUS is an acceptable form of contraception in young women, including nulliparous women, and should be offered alongside other methods as first line without restriction when offering contraceptive options to this age group.

\section{INTRODUCTION}

Despite the perception that there is widespread access to contraceptive advice and methods, unintended pregnancy remains a major health issue in the UK. ${ }^{1}$ Termination of pregnancy rates in England and Wales continue to rise. In 2010, the agestandardised rate was 17.5 per 1000 resident women aged 15-44 years, the same as in 2009 , but $3 \%$ higher than in 2000 (17.0) and more than double the rate of

\section{KEY MESSAGE POINTS}

- The levonorgestrel-releasing intrauterine system was an acceptable form of contraception for young women and nulliparous women in this study.

- There should be no restriction on this method of contraception being offered to these patient groups.

8.0 recorded in $1970 .^{2}$ In 2005 , the UK National Institute for Health and Clinical Excellence (NICE) issued guidelines advocating increased access to, and promotion of, long-acting reversible contraception (LARC) to impact on unplanned pregnancies. The levonorgestrel-releasing intrauterine system (LNG IUS; Mirena ${ }^{\circledR}$, Bayer) is one of a range of LARC methods available and is one of the most reliable and cost effective. $^{3}$ The Office for National Statistics Omnibus Survey 2007/8 showed that the majority of women questioned (74\%) were using a method of contraception. ${ }^{4}$ The most commonly used methods were oral contraception (28\%) and the male condom (24\%). Three percent of women questioned were using the IUS. Only $56 \%$ of 78 respondents aged 16-19 years questioned in this survey were using any method of contraception. A questionnaire-based survey of general practitioners (GPs) within one borough in London showed that nine out of ten GPs considered the contraceptive pill as the first choice for nulligravid women aged under 25 years, with the IUS being the least recommended LARC for this group of women. ${ }^{5}$ 


\section{METHODS}

The setting of this large university-based general practice offers the full range of contraceptive choices to all patients and has eight clinicians within the team who can fit intrauterine contraceptives (IUDs/IUS).

A consecutive series of 100 women attending for fitting of the IUS between 1 November 2009 and 22 July 2010 were questioned at the time of fitting on reason for choice. Demographics, pregnancy history, status for Chlamydia trachomatis and reason for choice were documented in a questionnaire completed by the clinician. Patient consent was obtained to allow data collection and follow up over the following 12 months. There were no specified inclusion or exclusion criteria. All 100 women agreed to take part and be followed up within this prospective observational study. Follow up by telephone was undertaken at 6 weeks, 6 months and 9-12 months after fitting to check satisfaction with method and continuation, or reason for discontinuation. Data were collated and analysed in Microsoft Excel ${ }^{\mathrm{TM}}$. A review of published literature was undertaken using PubMed with the search terms 'Mirena', 'levonorgestrel', 'IUS', 'young women' and 'adolescents'.

\section{RESULTS}

As of 4 May 2011, the practice had 35989 registered adult patients with 19948 adult female patients. The age range of female adult patients registered at the practice was $18-58$ (mean 23) years. The age range of patients within the study was $18-38$ years; this is normally distributed with a mean of 22 years and standard deviation of 2 years 5 months. Ninety-one patients were born in the UK.

Ninety-seven women were nulliparous with 95 women never having been pregnant.

Ninety-nine women had had a chlamydia test within 12 months of the fit, 94 of which were done at the practice. The mean time between testing and insertion was 37.7 (range 25-353) days. One result was positive.

The reasons for choice were varied. The figures that follow relate to the total group, with the figures in parentheses relating to nulliparous women. Forty (39) women chose the IUS as they were having problems with their current methods of contraception. Thirty-seven (37) women stated that the main reason for selecting the IUS was that it was their preferred choice for LARC. Six (6) women had a removal and reinsertion at 5 years' use. Twelve (10) women chose the IUS primarily for its non-contraceptive benefits. There were 31 medical students in the cohort (see Figure 1 relating to nulliparous women).

Of the 100 women, 75 (75) still had the device at 9-12 months with 11 lost to follow up at this point. Of these 11, two could not be contacted at all after insertion but nine women were contactable at 6 weeks to 6 months and still had their device in situ. Five of the 11 women left the practice during the study period. One attempted insertion was unsuccessful and three devices were expelled. Ten (9) devices were removed in total: five (4) due to complaints of pain, one (1) because it could be felt by the partner, one (1) in a woman who needed treatment for abnormal cervical cytology and one (1) in a woman who felt that the IUS was directly affecting her ability to concentrate. Two (2) devices were removed due to what the women felt was an unacceptable bleeding pattern (see Figure 2 relating to nulliparous women).

No pregnancies or perforations were reported. No removal was associated with symptoms of infection and no cases of suspected pelvic infection/pelvic inflammatory disease were reported. Forty-six copper IUDs were fitted in the practice during the recruitment period.

\section{DISCUSSION}

The results of our series support the fact that the IUS is an acceptable form of contraception in young,

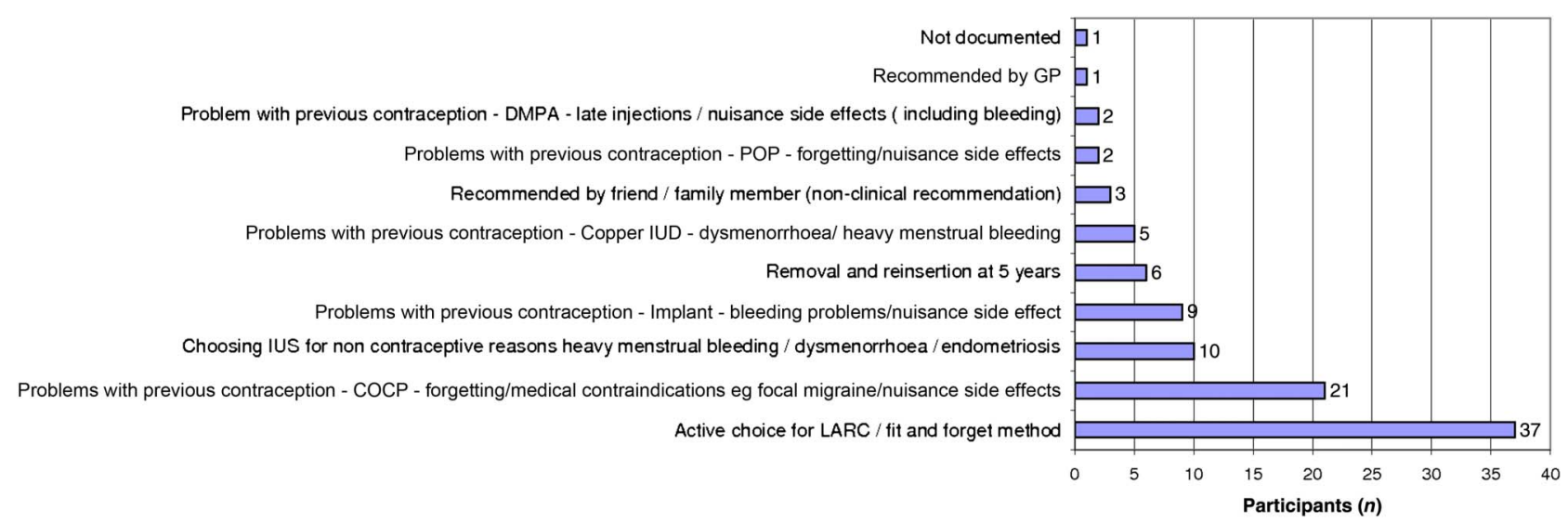

Figure 1 Main reason for choosing the intrauterine system (nulliparous women, $n=97$ ). COCP, combined oral contraceptive pill; DMPA, depot medroxyprogesterone acetate; GP, general practitioner; IUD, intrauterine device; IUS, intrauterine system; LARC, long-acting reversible contraception; POP, progestogen-only pill. 


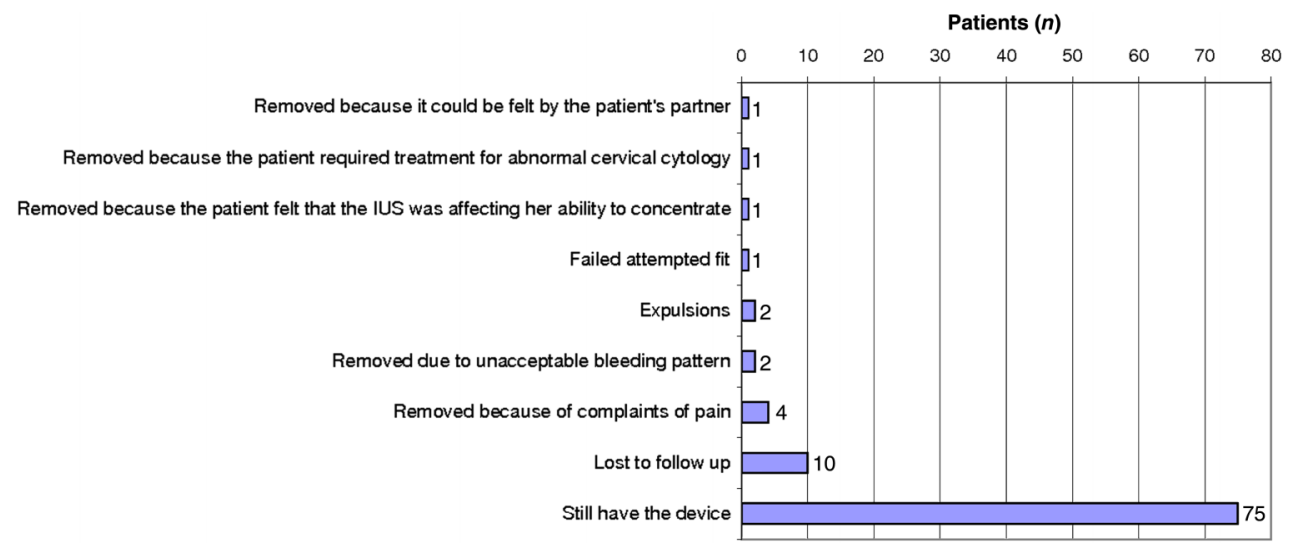

Figure 2 Outcome at 12 months' follow up/continuations (nulliparous women, $n=97$ ). IUS, intrauterine system.

nulliparous women and may be chosen in preference to other methods as first line.

On review of the articles found during the literature search, most study groups were not of comparable age and/or parity to our study population. ${ }^{6} 7$ The problem with definition of 'young women' and 'adolescent' means that it was hard to draw comparisons between studies. One double-blind randomised controlled trial in the USA had 12 women in the LNG IUS arm and the study acknowledged it was underpowered. ${ }^{8}$ One retrospective case note review set in New Zealand looked at 11-19-year-olds, $73 \%$ of whom were nulliparous at the time of insertion but $37 \%$ of all insertions were under general anaesthetic. ${ }^{9}$ A systematic review by USA authors of IUDs in adolescents did not find any studies on LNG IUS to meet its inclusion criteria. ${ }^{10}$

One randomised trial conducted in Sweden and Finland compared the clinical performance of the LNG IUS with oral contraceptives (OCs) in young women. All women were aged 18-25 years and were nulliparous. Nineteen of the 94 women in the IUS arm discontinued the study, mostly due to pain, but $27 / 99$ women in the OC arm also discontinued, mostly due to hormonal side effects. ${ }^{11}$ One USA-based review article quoted a 1-year continuation rate of 80 per 100 users amongst nulliparous women. ${ }^{12}$ An observational study in Sweden following nulliparous women with the IUS had a removal rate of $23.8 \%$ at 1 year but $38 \%(82 / 216)$ of women were lost to follow up. ${ }^{13} \mathrm{~A}$ retrospective cohort study of women fitted with an IUS in the USA during a 34-month period showed that adolescents and nulliparous women were no more likely to prematurely discontinue use of the IUS than adult or parous women. ${ }^{14}$ Figures from the Office of National Statistics in the UK show that $2 \%$ of 16-19-year-olds using contraception were using the IUS. Less than $1 \%$ of 20-24-year-olds were using the IUS. ${ }^{15}$

Myths associated with use of IUDs/IUS still exist despite guidance to the contrary. ${ }^{16}$ Clinicians may be concerned about associations with ectopic pregnancy, infertility and pelvic infection. There may also be hesitation in recommending use of IUDs/IUS in young women and in nulliparous women due to perceived difficulties with fitting. The UK Medical Eligibility Criteria for Contraceptive Use (UKMEC 2009) provides guidance for clinicians on appropriate use and safety of contraceptive methods. This includes a section on use of IUDs and there are very few situations labelled as UKMEC Category 4, where there is an unacceptable health risk if the method is used. ${ }^{17}$

\section{CONCLUSIONS}

There are few available data on the use of the IUS in young, nulliparous women in the UK. This study supports our impression that the IUS is very acceptable to young nulliparous women, and may increasingly be requested as an active choice for contraception, with young women appreciating the benefits of ' $f$ it and forget' contraception. There should be no barriers to access for this method for young women or nulliparous women. Age alone should not limit contraceptive choices, including intrauterine methods. Young people should be informed of, and offered, all methods of contraception, and the benefits of LARC methods highlighted. ${ }^{18}$

Acknowledgements The authors would like to thank Drs Farah Chaudhry, Natalie Hodgson, Julianne Lyons and Dawn Smith for their contributions.

Funding This investigator-sponsored study was supported financially by Bayer Healthcare Pharmaceuticals.

Competing interests Drs Catherine Armitage and Deborah Smith have received sponsorship and consultancy fees from Bayer Healthcare Pharmaceuticals.

Authors' note Interim data were presented as poster presentations at the European Society of Contraception Conference in 2010 and at the European Society of Gynaecology Conference in 2011.

Provenance and peer review Not commissioned; externally peer reviewed.

\section{REFERENCES}

1 Fleming CF. Review - long-acting reversible contraceptives. Obstetrician and Gynaecologist 2009;11:83-88. 
2 Department of Health. Abortion Statistics, England and Wales: 2007. http://www.dh.gov.uk/prod_consum_dh/groups/ dh_digitalassets/documents/digitalasset/dh_127202.pdf [accessed 28 December 2011].

3 National Institute for Health and Clinical Excellence (NICE). Long-acting Reversible Contraception. 2005. http://www.nice. org.uk/nicemedia/live/10974/29912/29912.pdf [accessed 28 December 2011].

4 Office for National Statistics. Contraception and Sexual Health 2007/08 (Omnibus Survey Report No. 37). 2008. http://www. ons.gov.uk/ons/rel/lifestyles/contraception-and-sexual-health/ 2007-08/2007-08.pdf [accessed 28 December 2011].

5 Middleton AJ, Naish J, Singer N. General practitioners' views on the use of the levonorgestrel-releasing intrauterine system in young nulligravid women, in London, UK. Eur J Contracep Reprod Health Care 2011;16:311-318.

6 Campbell SJ, Cropsey K, Matthews C. Intrauterine device use in a high-risk population: experience from an urban university clinic. Am J Obstet Gynecol 2007;197:193. e1-193.e7.

7 Baldaszti E, Wimmer-Puchinger B, Loschke K. Acceptability of the long term contraceptive levonorgestrel-releasing intrauterine system (Mirena ${ }^{\mathrm{TM}}$ ): 3 year follow up study. Contraception 2003;67:87-91.

8 Godfrey E, Memmel L, Neustadt A, et al. Intrauterine contraception for adolescents aged 14-18 years: a multicenter randomized pilot study of the levonorgestrel-releasing intrauterine system compared to the Copper T380A. Contraception 2010;81:123-127.

9 Paterson H, Ashton Harrison-Woolrych M, et al. A nationwide cohort study of the use of the levonorgestrel intrauterine device in New Zealand. Contraception 2009;79:433-438.
10 Deans E, Grimes D. Intrauterine devices for adolescents: a systematic review. Contraception 2009;79:418-423.

11 Suhonen S, Haukkamaa M, Jakobsson T, et al. Clinical performance of a levonorgestrel-releasing intrauterine system and oral contraceptives in young nulliparous women: a comparative study. Contraception 2004;69: 407-412.

12 Prager S, Darney P. The levonorgestrel intrauterine system in nulliparous women. Contraception 2007;75:S12-S15.

13 Marions L, Lovkvist L, Taube A, et al. Use of the levonorgestrel releasing intrauterine system in nulliparous women - a non-interventional study in Sweden. Eur J Contracept Reprod Health Care 2011;16:126-134.

14 Behringer T, Reeves M, Rossiter B, et al. Duration of use of a levonorgestrel IUS amongst nulliparous and adolescent women. Contraception 2011;84:e5-e10.

15 Office for National Statistics. Contraception and Sexual Health 2008/09. (Opinions Survey Report No. 41). 2009. http://www. statistics.gov.uk/downloads/theme_health/contra2008-9.pdf [accessed 3 April 2010].

16 Faculty of Sexual and Reproductive Health Care Clinical Effectiveness Unit. Intrauterine Contraception. 2007. http:// www.fsrh.org/pdfs/CEUGuidanceIntrauterineContraception Nov07.pdf [accessed 28 December 2011].

17 Faculty of Sexual and Reproductive Health Care. UK Medical Eligibility Criteria for Contraceptive Use. 2009. http://www. fsrh.org/pdfs/UKMEC2009.pdf [accessed 28 December 2011].

18 Faculty of Sexual and Reproductive Health Care Clinical Effectiveness Unit. Contraceptive Choices for Young People. 2010. http://www.fsrh.org/pdfs/ceuGuidanceYoungPeople2010. pdf [accessed 28 December 2011]. 